\title{
Management of an oncological wound: Diagnosis, conflicting therapies and decision-making
}

\author{
This article presents the case of a non-HIV-positive patient who \\ contemporaneously suffered from Kaposi's sarcoma and bullous pemphigoid. \\ Kaposi's sarcoma is a rare low-grade vascular tumour associated with \\ human herpes virus 8 infection, while bullous pemphigoid is the most \\ common autoimmune subepidermal blistering \\ disease in western countries. \\ Keywords: \\ bullous pemphigoid; kaposo sarcoma; chronic wound
}

\section{INTRODUCTION}

Kaposi's sarcoma (KS) is a rare low-grade vascular tumour associated with human herpes virus 8 infection (HHV-8). ${ }^{1}$ Bullous pemphigoid (BP) is the most common autoimmune subepidermal blistering disease in western countries, and typically affects the elderly. ${ }^{2}$ We present the case of a non-HIV-positive patient who contemporaneously suffered from Kaposi's sarcoma and bullous pemphigoid.

Oncological wounds are often large, disfiguring, exuding, malodorous and hard to manage. However, while many oncological wounds do not have these properties, their diagnosis and management can still pose a challenge. For example, diagnosis of Kaposi's sarcoma or epithelial skin tumours is particularly difficult because they are often mistaken for common types of chronic wounds, such as venous leg ulcers or diabetic foot ulcers.

\section{CASE REPORT}

A 94-year-old male patient was referred to our dermatological Wound Care Clinic at the University Hospital of Bologna for a wound on the plantar area of his right foot.

The lesion, measuring $12 \times 8 \mathrm{~cm}$, had been present for a few months and was progressively worsening. The patient had previously been managed by the district nurses.

The patient suffered from diabetes mellitus type 2 with mild symptoms of diabetic neuropathy, dyslipidaemia, chronic obstructive pulmonary disease, ischaemic heart disease, hypertension, hyperthyroidism and benign prostatic hypertrophy.

The patient's medical history included a recent BP diagnosis, for which he had been treated with systemic 


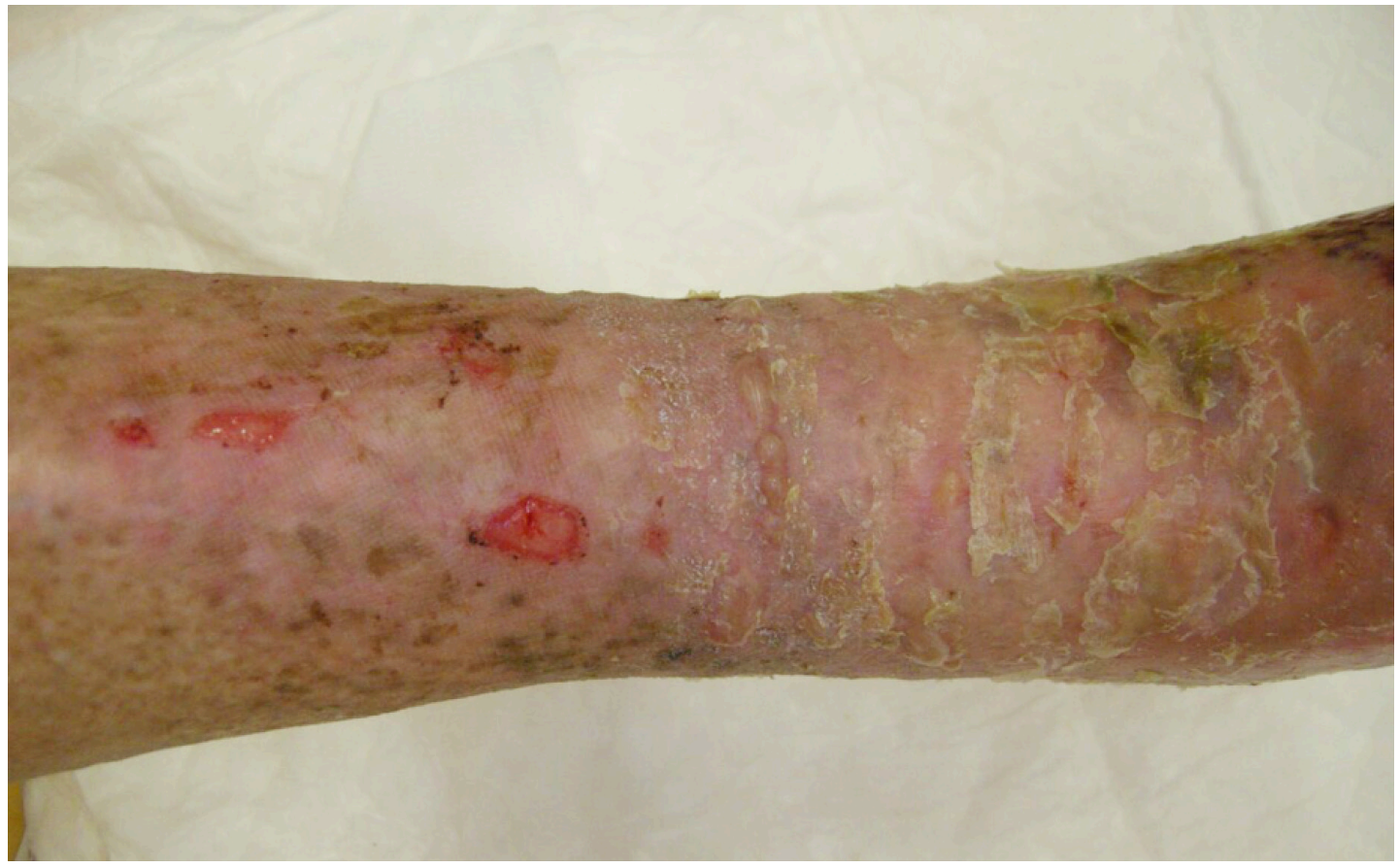

Figure 1. At admission - state of the leg and Bullous Pemphigoid eroded blisters after 2 months of immunosuppressive treatment.

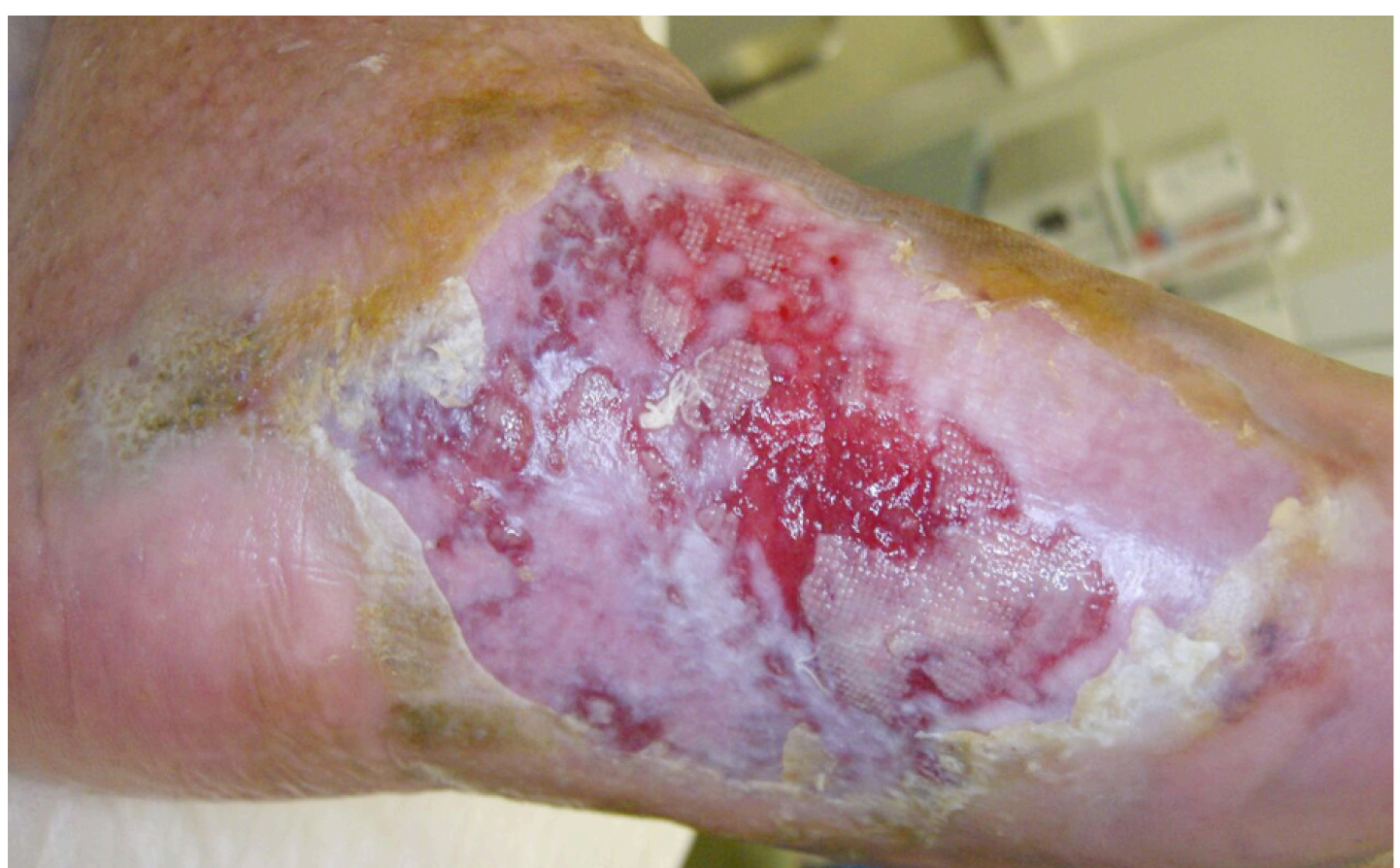

Figure 2. At admission - wound located in plantar area of left foot: hyperplastic violaceous granulation tissue suggesting for Kaposi Sarcoma.

corticosteroids for 2 months, and relapsing episodes of small leg ulcers resulting from eroded blisters connected to BP (Fig. 1), which had completely regressed after systemic immunosuppressive therapy. The plantar lesion had been present for about 3 months, but it was non-healing and it was deteriorating.

The patient could still walk and carry out the main activities of daily life independently, using orthopae- dic pressure relieving shoes and a walking stick. He complained of not being able to move as freely as he used to due to the large amount of exudate and the frequent dressing changes. The patient lived in a retirement home with the support of his two sons, who accompanied him to the Wound Care Clinic for dressing changes and medical visits.

On examination, the wound presented heavily ex- 


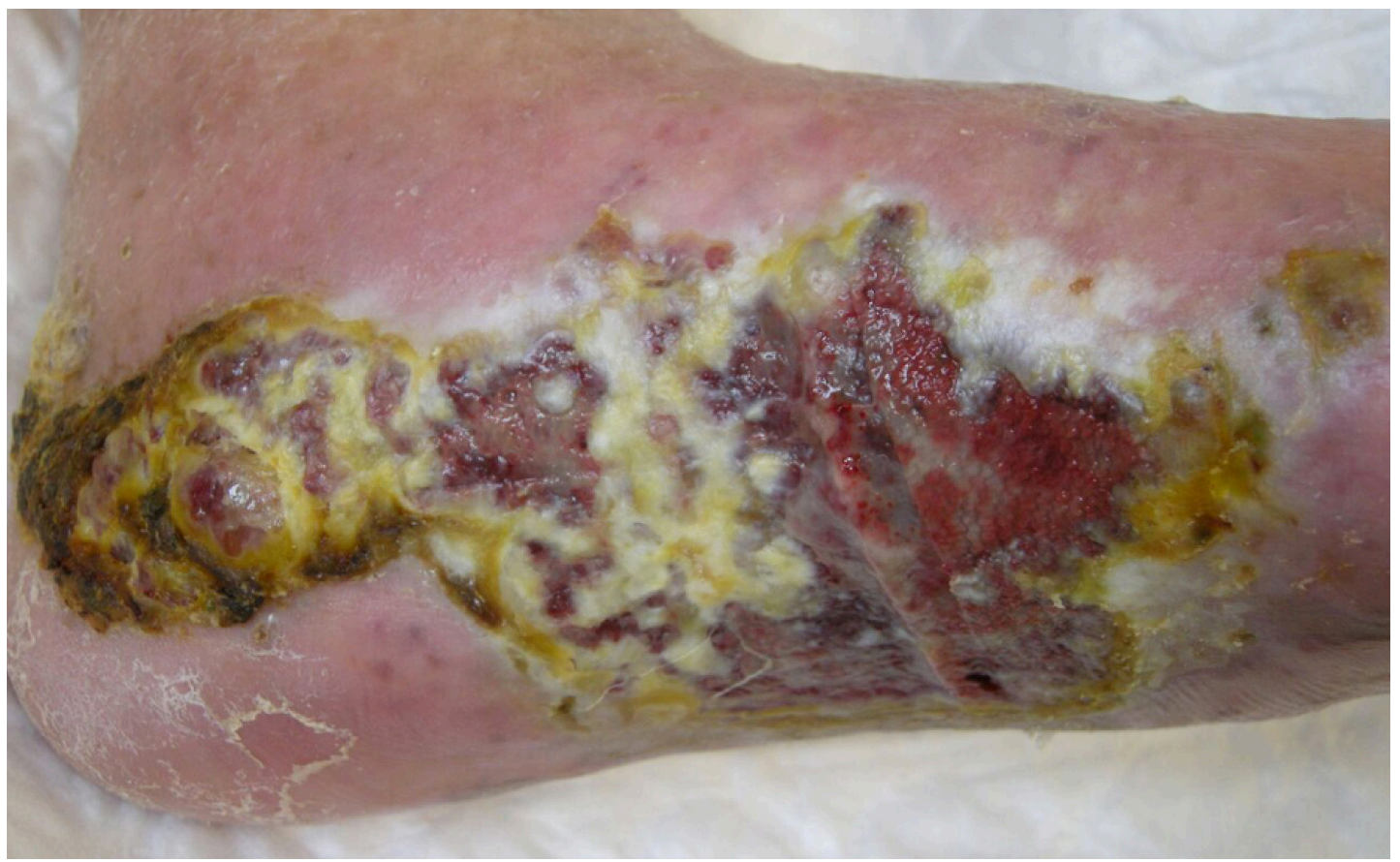

Figure 3. State of the wound 14 months into the treatment. Worsening of the KS is evident. Wound with hyperkeratotic necrotic swelling linked to the tumor.

uding, violaceous granulation tissue and macerated hyperkeratotic borders (Fig. 2). The right leg was oedematous.

Skin swabs were carried out in order to profile the cutaneous microbiota and perform antibiotic-susceptibility tests. Once the skin swabs were executed, the patient was treated for his wounds with alginatebased dressings containing silver and multi-layer compressive bandaging.

A week later the swabs showed no signs of infection. However, despite wound management with advanced dressings twice a week, no resolution or improvement was detected after a month's therapy. Due to the wound's chronic nature, a skin biopsy for histopathological examination was performed. It revealed an increased number of atypical vascular structures with thinned walls and proliferating bundles of spindle endothelial cells. The clinical picture, added to the histological features, suggested KS as a possible diagnosis. Complementary immunohistochemistry was carried out and the HHV-8 positivity confirmed the diagnosis.

The screening exams performed to exclude systemic involvement, including chest X-ray, abdominal ultrasound and digestive endoscopy, were all negative. Therefore, an oncological consultation was promptly requested. Following a team meeting, conservative palliative therapy was chosen because of the patient's multiple comorbidities and advanced age. The treatment consisted of exudate management and bioburden control. BP was treated by alternating short cycles of systemic steroids with topical steroid-based ointments.

The patient is currently in the care of our outpatient Wound Care Clinic. More than one year after the KS diagnosis, the clinical picture has progressively worsened (Fig. 3).

The patient maintained a good degree of autonomy in his activities of daily living for about 4 months. Afterwards the patient was no longer able to ambulate independently, although he is still able to walk indoors with the aid of a walker. The patient is still in good cognitive condition, scoring 26 points on the Mini-Mental State Examination. ${ }^{3}$

\section{DISCUSSION}

Kaposi's sarcoma risk is increased in immunocompromised patients, including those undergoing immunosuppressive therapy for autoimmune bullous diseases. Conversely, HHV-8 infection has been hypothesised to be a triggering factor of bullous diseases, especially pemphigus. ${ }^{4}$

The main goals of KS therapy are symptom palliation, prevention of disease progression and tumour 
shrinkage. Local treatments, including radiation therapy, intra-lesional chemotherapy and topical alitretinoin, can manage symptomatic bulky KS lesions or achieve better cosmetic results, but they do not prevent disease progression. ${ }^{5}$ Systemic treatment with chemotherapy, liposomal anthracyclines, taxanes and anti-HHV-8 therapy are used in cases of advanced disease or rapid tumour progression. ${ }^{6}$

$\mathrm{BP}$ is an autoimmune disease that can be triggered by drugs such as diuretics, antiarrythmics-antihypertensives, antitumor necrosis factor agents, antibiotics and worsened by physical stimuli, including radiotherapy. ${ }^{7} \mathrm{BP}$ treatment includes mainly immunosuppressive systemic therapies. ${ }^{2}$

The treatment of choice for KS, in this case, would have been radiotherapy, which could have worsened BP. However, for BP, the therapy of choice, systemic immunosuppressive drugs, would have led to a higher risk of infection and potentially a faster KS progression. People with oncological wounds suffer from a devastating symptom burden, such as pain and discomfort, irritation or itching, exudate, odour, infection and slough or necrosis; due to the chronic nature of the condition, deterioration is inevitable. ${ }^{8-10}$ In this patient's case, currently, most of the wound bed is covered with non-vitalised tissue, and the surrounding skin is hyperkeratotic with swelling linked to the tumour. However, the wound is free of infection. The right treatment approach is to take into account the patient and family's goals of care in all aspects of decision-making. The need for balance between the disease treatment and the patient's need for autonomy and less frequent dressing changes motivated our clinical intervention. The frequency of the dressing changes depends still on the exudate and inflammation, but our objective is to find a way to reduce them. For the team, it is frustrating to witness the worsening of the wound and be unable to heal it.

\section{CONCLUSION}

The patient was affected by two debilitating chronic diseases, for which the treatment for each one worsened the condition of the other. A difficult clinical decision had to be made. From the holistic evaluation of the patient and his family it became apparent that quality of life was the most important factor to take into consideration. Therefore, conservative therapy was undertaken.

BP was treated using short cycles of systemic steroids that were then alternated with topical steroids only, while the lesion caused by KS was managed using antimicrobial dressings and multi-layer compression bandaging to address the exudate, the risk of infection and possibly pain and to contain the oedema.

In this case the additional presence of BP, which could present with erosions and ulcers, was a confounding factor.

The primary obstacle for this patient was the delay in having a correct diagnosis. In this case it took around two months before the patient was referred to a specialised centre and another month before we carried out the biopsy confirming the oncological aetiology of the wound.
REFERENCES

1. Goncalves PH, Ziegelbauer J, Uldrick TS, Yarchoan R. Kaposi sarcoma herpesvirus-associated cancers and related diseases. Curr Opin HIV AIDS. 2017 Jan;12(1):47-56.

2. Bernard P, Antonicelli F. Bullous pemphigoid: a review of its diagnosis, associations and treatment. Am J Clin Dermatol. 2017 Aug; 18(4):513-528.

3. Ghafar MZAA, Miptah HN, O'Caoimh R. Cognitive screening instruments identify vascular cognitive impairment: A systematic review. Int J Geriatr Psychiatry. 2019 Aug.34(8):1114-1127. do: 10.1002/gps.5136. Epub 2019 May 16.
4. Tourlaki A, Genovese G, Guanziroli E, Scoppio BM, Berti E, Brambilla L. Autoimmune bullous diseases in non-HIV Kaposi's sarcoma: a retrospective study in a large cohort of patients. J Eur Acad Dermatol Venereol. 2018 Oct;32(10):1777-1783.

5. Phavixay L, Raynolds D, Simman R. Non AIDS Kaposi's sarcoma leading to lower extremities wounds, case presentations and discussion. J Am Coll Clin Wound Spec. 2013 Jan 19;4(1):13-15.

6. Cesarman E, Damania B, Krown SE, Martin J, Bower M, Whitby D. Kaposi sarcoma. Nat Rev Dis Primers. 2019 Jan 31;5(1):9.

7. Kluger N, Mandelin J, Santti K, Jeskanen L, Nuutinen P. Bullous pemphigoid triggered by radiotherapy for breast cancer. Presse Med. 2017 Jan;46(1):128-130.
8. Tilley C, Lipson J, Ramos M. Palliative wound care for malignant fungating wounds: holistic consideration at end-of-life. Nurs Clin North Am. 2016 Sep;51(3):513-531.

9. Probst S, Arber A, Faithfull S. 2013 Malignant fungating wounds: the meaning of living in an unbounded body. Eur J Oncol Nurs. 2013 Feb;17(1):38-45.

10. Probst S, Arber A, Trojan A, Faithfull S. Coping with an exulcerated breast carcinoma: an interpretative phenomenological study. J Wound Care. 2013 Jul;22(7):352-354, 356-358, 360. 